\title{
A review of medication
} incidents reported to the National Reporting and Learning System in England and Wales over 6 years (2005-2010)

\author{
David H. Cousins, David Gerrett \& Bruce Warner
}

National Patient Safety Agency, London, UK

\section{Correspondence}

Dr David Cousins BPharm, MSc, PhD, FRPharmS, National Patient Safety

Agency, London W1T 5HD, UK.

Tel.: 447968288098

Fax: 442079279501

E-mail david.cousins@npsa.nhs.uk

Keywords

errors, medication incidents, national patient safety agency, patient safety

Received

26 September 2011

Accepted

15 December 2011

Accepted Article

Published Online

22 December 2011

A review of all medication incidents reported to the National Reporting and Learning System (NRLS) in England in Wales between 1 January 2005 and 31 December 2010 was undertaken. The 526186 medication incident reports represented $9.68 \%$ of all patient safety incidents. Medication incidents from acute general hospitals (394 951) represented $75 \%$ of reports. There were relatively smaller numbers of medication incident reports (44 952) from primary care, representing $8.5 \%$ of the total. Of 86821 (16\%) medication incidents reporting actual patient harm, $822(0.9 \%)$ resulted in death or severe harm. The incidents involving medicine administration (263 228; 50\%) and prescribing $(97097 ; 18 \%)$ were the process steps with the largest number of reports. Omitted and delayed medicine $(82028 ; 16 \%)$ and wrong dose $(80170 ; 15 \%)$ represented the largest error categories. Thirteen medicines or therapeutic groups accounted for 377 (46\%) of the incidents with outcomes of death or severe harm. The National Patient Safety Agency (NPSA) has issued guidance to help minimize incidents with many of these medicines. Many recent incidents could have been prevented if the NPSA guidance had been better implemented. It is recommended that healthcare organizations in all sectors establish an effective infrastructure to oversee and promote safe medication practice, including an annual medication safety report. In the future, preventable harms from medication incidents can be further minimized by; the continued use of the NRLS to identify and prioritize important actions to improve medication safety, a central organization continuing to issue medication safety guidance to the service and better methods to ensure that the National Health Service has implemented this guidance.

\section{Introduction}

In the report entitled 'An organisation with a memory' published in 2001, the Department of Health in England recognized that preventable harms were occurring in the National Health Service (NHS) and more could be done to minimize these harms [1]. The report identified two main areas where the NHS could draw valuable lessons from the experience of other sectors to minimize preventable harms. The first area was safety culture, where open reporting and balanced analysis are encouraged in principle and by example, which can have a positive and quantifiable impact on the recognition and management of preventable harms. The second area was reporting systems, which were considered vital in providing a core of sound, representative information on which to base analy- sis and recommendations. Experience in other sectors demonstrates the value of systematic approaches to recording and reporting adverse events and the merits of using information on 'near misses' as well as events that actually result in harm.

In order to better address the problem of patient safety in the NHS, The National Patient Safety Agency (NPSA) was established as a Special Health Authority in the NHS in England and Wales in 2001 [2]. The agency has been responsible for the National Reporting and Learning System (NRLS) that collects, analyses and learns from all types of patient safety incidents (PSIs). A patient safety incident has been defined by the NPSA as any unintended or unexpected incident which could have or did lead to harm for one or more patients receiving NHS-funded healthcare. 
In 2010, the UK government published plans to reduce NHS administrative costs by more than $45 \%$ and to simplify and radically reduce the number of NHS bodies in England [3]. The government's plans are intended to better align NHS bodies with the rest of the health and social care system by ensuring that functions related to quality and safety improvement are devolved closer to the front line and by integrating and streamlining existing functions. These proposals include that the NPSA will be abolished [3]. It is proposed that the NPSA ceases as a legal entity in July 2012 and that the responsibility for the NRLS will be transferred to a new organization, The NHS Commissioning Board [3].

The NRLS was rolled out during 2003, and by 1 January 2005 all NHS organizations in England and Wales were connected to the system and had the ability to report PSIs.

The aim of this review, prior to the closure of the NPSA, is to summarize the medication practice activities of the Agency to inform others with an interest in promoting the safe use of medicines in the future. Our review describes how many medication incidents, of what type and with what clinical outcomes have been reported, what learning and changes in practice have resulted from this information to make the use of medicines safer, and how processes for reporting and learning of medication incidents can be improved in the future.

The NPSA has previously published two reports of quantitative and qualitative data on medication incidents for periods of up to 18 months $[4,5]$. The present report, however, presents a larger and more extensive review of medication incident reports over a 6 year period.

\section{Methods}

All incidents reported as occurring between 1 January 2005 and 31 December 2010 were extracted from the NRLS database on 12 May 2011 using a SAS ${ }^{\circledR}$ Enterprise Guide 4.3 add-on for Microsoft Office Excel ${ }^{\circledR}$ and a medication-specific map of the NRLS, PSI data.

All reports with outcomes of death and severe harm were reviewed by an author (DG). Where necessary, the clinical outcome codes were changed to reflect more accurately the details of the reported incident. The definition of severe harm used for this paper was 'a patient safety incident that appears to have resulted in permanent harm and/or a near death experience.' Patient safety incidents were considered not applicable if they were adverse drug reactions where the harm was not avoidable, the PSI was miscoded or there was insufficient information to make any judgement of clinical outcome. To provide an understanding of the variation and amount of information describing reports of deaths and severe harm, this data set was investigated for the number of words reported, or not, in the categories'description of what happened','actions preventing reoccurrence' or 'apparent causes'. The results for deaths and severe harm were then compared with all medication reports in these categories for the 12 month period to 31 December 2010, to indicate whether severity of harm has any bearing on the extent of information provided.

A second data set of all NRLS updated PSI reports was extracted on 30 June 2011 using the SAS ${ }^{\circledR}$ Enterprise Guide 4.3 outputting directly to Excel ${ }^{\circledR}$ pivot tables. The NRLS is a dynamic database, where NHS organizations are able to upload, update and amend PSIs retrospectively. The tendency is for numbers to decrease as PSIs, for example, reported in error or duplicated are removed. This accounts for the small decrease in medication PSIs for the later extraction.

Where comparisons are drawn between medication and total PSIs, the second extraction is used (Tables 1-4). Raw data from the earlier extraction forms the basis of more in-depth analysis of medication PSIs (Tables 5-8).

Validation of patient-harm reclassification for the medication-only PSI data set was performed using a random sample of 10 deaths and severe harm PSIs from each of the 6 years. The 60 clinical outcomes were independently reviewed by the second author and compared.

Analysis was undertaken with SPSS version 17. Significance was determined at the $95 \%$ confidence interval. Regression analysis used the 'enter' model.

\section{Results}

Of the 5437999 total PSIs over the 6 years from 2005 to 2010, extracted in June 2011 (Table 1), 526186 medication incidents were reported. There has been a significant and consistent increase of over half a per cent each year in reported medication incidents relative to total PSIs [Table 1; percentage medication incidents of total reports by year, $\left.r^{2}=0.98, \beta=0.55(0.44-0.66)\right]$. Reports of medication incidents increased significantly by over 17000 each year [Table 1; medication incidents by year, $r^{2}=0.98$,

\section{Table 1}

Number of patient safety incidents and medication incidents received by the NRLS, 2005-2010*

\begin{tabular}{|lcll|}
\hline Year & $\begin{array}{l}\text { Total number } \\
\text { of incident } \\
\text { reports }\end{array}$ & $\begin{array}{l}\text { Number of } \\
\text { medication } \\
\text { incident } \\
\text { reportst }\end{array}$ & $\begin{array}{l}\text { Medication } \\
\text { incident reports as } \\
\text { a percentage of all } \\
\text { incident reports received }\end{array}$ \\
\hline $\mathbf{2 0 0 5}$ & 517415 & 42398 & 8.19 \\
\hline $\mathbf{2 0 0 6}$ & 742418 & 64484 & 8.69 \\
\hline $\mathbf{2 0 0 7}$ & 874148 & 79118 & 9.05 \\
\hline $\mathbf{2 0 0 8}$ & 986981 & 94280 & 9.55 \\
\hline $\mathbf{2 0 0 9}$ & 1118336 & 113837 & 10.18 \\
\hline $\mathbf{2 0 1 0}$ & 1198701 & 132069 & 11.02 \\
\hline Total & 5437999 & 526186 & - \\
\hline
\end{tabular}

*Based on the June 2011 extraction. 


\section{Table 2}

Categories of incidents reported to the NRLS, 2005-2010*

\begin{tabular}{|lrc|}
\hline Incident category & $\begin{array}{l}\text { Number of } \\
\text { incidents }\end{array}$ & $\begin{array}{l}\text { Percentage of all } \\
\text { incident reports }\end{array}$ \\
\hline Patient accident & 1785537 & 32.83 \\
\hline Medication & 526186 & 9.68 \\
\hline $\begin{array}{l}\text { Treatment/procedure } \\
\text { Access, admission, transfer and discharge, } \\
\text { missing patients }\end{array}$ & 434265 & 9.59 \\
\hline $\begin{array}{l}\text { All other types of incidents } \\
\text { Total }\end{array}$ & 7.99 \\
\hline
\end{tabular}

*Based on the June 2011 extraction.

\section{Table 3}

Number of patient safety incident reports and medication incidents received by the NRLS from each healthcare sector*

\begin{tabular}{|c|c|c|c|}
\hline Healthcare sector & $\begin{array}{l}\text { Total } \\
\text { number of } \\
\text { incident } \\
\text { reports }\end{array}$ & $\begin{array}{l}\text { Number of } \\
\text { medication } \\
\text { incident } \\
\text { reports }\end{array}$ & $\begin{array}{l}\text { Medication } \\
\text { incidents as a } \\
\text { percentage of } \\
\text { total incident } \\
\text { reports }\end{array}$ \\
\hline Acute/general hospital & 3921212 & 394951 & 10.07 \\
\hline Mental health service & 754812 & 48951 & 6.49 \\
\hline $\begin{array}{l}\text { Community nursing, medical } \\
\text { and therapy service } \\
\text { (including community } \\
\text { hospital) }\end{array}$ & 542323 & 48594 & 8.96 \\
\hline Learning disabilities service & 155914 & 8154 & 5.23 \\
\hline General practice & 22587 & 5358 & 23.72 \\
\hline Community pharmacy & 19696 & 19245 & 97.71 \\
\hline Ambulance service & 18415 & 712 & 3.87 \\
\hline $\begin{array}{l}\text { Community and general } \\
\text { dental service }\end{array}$ & 2560 & 133 & 5.20 \\
\hline $\begin{array}{l}\text { Community } \\
\text { optometry/optician service }\end{array}$ & 82 & 4 & 4.88 \\
\hline Not stated & 398 & 84 & 21.11 \\
\hline Total & 5437999 & 526186 & 9.68 \\
\hline
\end{tabular}

*Based on the June 2011 extraction.

$\beta=17474$ (16 116-18 831)]. Total PSI reports increased by nearly 133000 each year [Table 1 ; total incidents by year, $r^{2}=0.98, \beta=132771$ (103 855-161 688)].

The 526186 medication incidents represented 9.68\% of all incidents (Table 2), which was the second highest category in the NRLS.

While the 5358 mediation reports from general practice was a small number in comparison with all medication reports (1.02\%; Table 3$)$, it is notable that it represented a high proportion $(23.72 \%)$ of all incident reports from general practice reports.

The acute sector reported the largest number of medication incidents (394951; 75\%). There were far fewer reports from primary care (44952; 8.5\%). Acute sector organizations had median values of between 130 and 937 medication incident reports each year, depending on the size of the organization (Table 4). By inspection within each acute organization size cluster, the highest reporting organizations had double the number of medication incident reports compared with similar organizations in the lower quartile.

Based on the May 2011 extraction data set, an analysis of clinical outcomes of medication incidents (Table 5) demonstrates that 86821 (16\%) of reported incidents have outcomes of actual patient harm. Of these, $822(0.15 \%)$ resulted in serious harm or death to the patient. Based on the same data set, the number of incidents arising from each step of the medicine use process is presented (Table 6). Large numbers of administration incidents (263 228; 50\%) were reported, followed by prescribing incidents $(97097 ; 18 \%)$. This can be explained, because there are many more medicines administered in hospitals each day compared with the number of medicines prescribed, so there are more opportunities for error at this stage of the process than for prescribing.

The number of incident reports for each type of medication error is presented in Table 7, where omitted and delayed medicine $(82028 ; 16 \%)$ and wrong dose (80 170; $15 \%)$ were the largest identified categories. The 'other' classification was used when a medication incident could not be classified using the NRLS categories, or where the reporters did not select a classification category that could have been used to describe the incident, or categories in local risk management systems were not mapped to NRLS classification codes.

A review of the most common medicines or therapeutic groups involved in incident reports with death and severe harm as outcomes is presented in Table 8, where the unsafe use of 13 medicines or therapeutic groups represents 377 $(46 \%)$ of all incidents with serious outcomes. Where there were incidents involving more than one medicine in a therapeutic group, the name of the group has been used. Where incidents only involved one medicine in a therapeutic group, the name of the medicine has been used.

Clinical validation of the May 2011 medication-only data set resulted in a reduction of 74\% (from 2156 to 551) in PSIs assigned to severe harm and 14\% (from 314 to 271) in level-of-harm death.

A $\kappa$ value of 0.517 (SEM 0.095) demonstrated moderate agreement between assessors assigning 60 randomly selected cases to the NPSA categories of patient harm with the specific definition of severe harm described in this paper. Eleven of the 60 randomly selected incident reports were allocated a fatal clinical outcome code by the primary reviewer. The second reviewer agreed on a fatal outcome code in nine of the 11 cases. There was a tendency for the main reviewer (D.G.) to assign comparatively greater numbers to the severe category of permanent harm. The implication is that the already large downward classification of reported severe harm is likely to be a conservative estimate. 


\section{Table 4}

Medication incidents reported by the acute care cluster type*

\begin{tabular}{|lllll|}
\hline Acute organization cluster & $\begin{array}{l}\text { Number of } \\
\text { reporting } \\
\text { organizations }\end{array}$ & $\begin{array}{l}\text { Acute organizations } \\
\text { that have not reported } \\
\text { any medication incidents }\end{array}$ & Lower quartile & $\begin{array}{l}\text { Upper } \\
\text { quartile }\end{array}$ \\
\hline Acute specialist trust & 20 & 1 & 31 & 291 \\
\hline Acute teaching trust & 25 & 0 & 164 & 131 \\
\hline Large acute trust & 44 & 0 & 150 & 813 \\
Medium acute trust & 51 & 1 & 112 & 376 \\
\hline Small acute trust & 30 & 1 & - & 265 \\
\hline Total & 170 & 3 & & 203.5 \\
\hline
\end{tabular}

*Based on the June 2011 extraction.

\section{Table 5}

Medication incidents reported by clinical outcome*

\begin{tabular}{|lcc|}
\hline Actual clinical outcome & Incidents & $\begin{array}{l}\text { Percentage of } \\
\text { medication incidents }\end{array}$ \\
\hline Death & 271 & 0.05 \\
Severe & 551 & 0.10 \\
Moderate & 17421 & 3.31 \\
Low & 68578 & 13.03 \\
No harm & 439318 & 83.46 \\
Not applicable & 240 & 0.05 \\
Total & 526379 & 100.00 \\
\hline
\end{tabular}

*Based on the May 2011 extraction following clinical validation for deaths and severe harm.

\section{Table 6}

Medication incidents by stage of medication process*

\begin{tabular}{|lrc|}
\hline Stage of medication process & Incidents & $\begin{array}{l}\text { Percentage of } \\
\text { medication } \\
\text { incidents }\end{array}$ \\
\hline Administration of medicines & 263228 & 50.01 \\
\hline Prescribing of medicines & 97097 & 18.45 \\
\hline Preparation/dispensing of medicines & 87057 & 16.54 \\
\hline Other & 48410 & 9.20 \\
\hline Monitoring/follow-up of medicine use & 23648 & 4.49 \\
\hline Advice & 3537 & 0.67 \\
\hline Supply or use of over-the-counter medicine & 3045 & 0.58 \\
\hline Not applicable & 240 & 0.05 \\
\hline Blank & 117 & 0.02 \\
\hline Other/unspecified & 48410 & 9.20 \\
\hline Total & 526379 & 100.00 \\
\hline
\end{tabular}

*Based on the May 2011 extraction following clinical validation for deaths and severe harm.

In the May 2011 extraction, of the 822 clinically validated PSIs with death or severe harm outcomes, the mean number of words in the category 'description of what happened' was just over 89 , with a median of $67\left(25^{\text {th }}\right.$ and 75th percentiles 43 and 106, respectively; range 8-711),

\section{Table 7}

Medication incidents by category of error reported*

\begin{tabular}{|lcc|}
\hline Category of error & Incidents & $\begin{array}{l}\text { Percentage of } \\
\text { medication } \\
\text { incidents }\end{array}$ \\
\hline Omitted and delayed medicine & 82028 & 15.58 \\
\hline Wrong dose or strength & 80170 & 15.23 \\
\hline Wrong medicine & 48834 & 9.28 \\
\hline Wrong frequency & 44165 & 8.39 \\
\hline Wrong quantity & 28764 & 5.46 \\
\hline Mismatching between patient and medicine & 21915 & 4.16 \\
\hline Wrong/transposed/omitted medicine label & 13755 & 2.61 \\
\hline Patient allergic to treatment & 11695 & 2.22 \\
\hline Wrong formulation & 11254 & 2.14 \\
\hline Wrong/omitted/passed expiry date & 10998 & 2.09 \\
\hline Wrong storage & 10447 & 1.98 \\
\hline Unknown & 10024 & 1.90 \\
\hline Wrong method of preparation/supply & 9840 & 1.87 \\
\hline Wrong route & 7934 & 1.51 \\
\hline Contra-indication to the use of the medicine & 7632 & 1.45 \\
\hline Adverse drug reaction (when used as & 5939 & 1.13 \\
\hline intended) & & \\
\hline Wrong/omitted verbal patient directions & 1383 & 0.26 \\
\hline Wrong/omitted patient information leaflet & 1156 & 0.22 \\
\hline Blank & 129 & 0.02 \\
\hline Other/not specified & 118317 & 22.48 \\
\hline Total & 526379 & 100.00 \\
\hline & & \\
\hline Based on the May 2011 extrattion folowing & & \\
\hline
\end{tabular}

*Based on the May 2011 extraction following clinical validation for deaths and severe harm.

implying a skewed distribution with a few very detailed reports (skewness +2.785 ). There were no words at all for the category'actions preventing reoccurrence' or'apparent causes' in 65.09 and $86.25 \%$ of reports, respectively, and in $62.53 \%$ of reports neither category contained any further information.

Similar results were found with the 132243 medication safety incidents for 2010 . There was no information in the categories 'actions preventing recurrence' $(60.05 \%)$ or 'apparent causes' (82.28\%), and in 59\% of reports neither category contained any information. Only $40 \%$ of reports 


\section{Table 8}

Medicines/therapeutic groups identified in incident reports with clinical outcomes of death and severe harm*

\begin{tabular}{|lrrrr|} 
& & & & $\begin{array}{l}\text { Percentage of } \\
\text { medication } \\
\text { incidents with } \\
\text { fatal and severe } \\
\text { harm outcomet }\end{array}$ \\
\hline Medicine or therapeutic group & Death & Severe & Total & \\
\hline Opioids & 46 & 43 & 89 & 10.83 \\
\hline Antibiotics & 10 & 38 & 48 & 5.84 \\
\hline Warfarin & 15 & 30 & 45 & 5.6 \\
\hline Low molecular weight heparin & 23 & 23 & 46 & 5.6 \\
\hline Insulin & 9 & 37 & 46 & 5.6 \\
\hline Benzodiazepines & 15 & 12 & 27 & 3.28 \\
Nonsteroidal & 1 & 17 & 18 & 2.19 \\
$\quad$ anti-inflammatory drugs & & & & \\
\hline Potassium & 7 & 8 & 15 & 1.82 \\
\hline Adrenaline & 8 & 4 & 12 & 1.46 \\
\hline Phenytoin & 1 & 11 & 12 & 1.46 \\
\hline Amiodarone & 3 & 4 & 7 & 0.85 \\
\hline Antipsychotics & 2 & 5 & 7 & 0.85 \\
\hline Methotrexate & 2 & 3 & 5 & 0.61 \\
\hline Total & 142 & 235 & 377 & 45.99 \\
\hline
\end{tabular}

*Based on the May 2011 extraction following clinical validation for deaths and severe harm.

tPercentage of the 822 total of deaths plus severe harm patient safety incidents.

identified the name of a medicine in the NRLS 'medicine name' data field. The name of the medicine, however, was often included in the free text describing the incident.

\section{Discussion}

\section{Number of incidents reported}

The increasing number of medication reports each year is significantly more than increases in the total number of patient safety incident reports to the NRLS. An improving safety culture within the NHS, where staff are more aware of patient safety and incident reporting, as well as being willing to report incidents within a fair blame culture, is the most likely explanation for the increase in the number of all PSI reports. The proportionally larger increase for medication incidents may be linked, in part, to increasing use of medicines in the NHS $[6,7]$.

It is disappointing that there are low numbers of PSI reports of all types from primary care. The improvement in the reporting culture seen in other healthcare sectors was not seen in this sector. It is recommended that alternative strategies are sought to improve reporting and learning of patient safety incidents from this sector. This is vital if improvements to safety across the whole system are to be both prioritized and realized.

Published studies indicate that between 1 and $10 \%$ of all prescribing [8-11], dispensing [12-14] and medicine administration [15-20] procedures in the UK include errors. Administration of intravenous medicines procedures have been found to have a higher error rate of $49 \%$ [21]. With thousands of these procedures occurring in NHS provider organizations each day, it is disappointing that there were individual acute sector organizations reporting less than 1000 medication incidents to the NRLS each year. A small number organizations did not report any medication incidents. Without reports, there can be no learning. While it is recognized that not all medication errors actually cause or have the potential to cause harm, these data indicate that there continues to be an under-reporting of medication incidents and that many more medication incidents are occurring than are reported each year. Barber et al. quantified the percentage of medication errors identified by spontaneous reporting as less than $1 \%$ of the total [20]. Regular local feedback to front-line staff indicating the number of medication incidents reported and the learning and system improvements derived from reports can help to increase the number of reports.

\section{Data quality}

The NRLS uses a data structure similar to that proposed by the World Health Organization International Classification for Patient Safety (ICPS) [22]. Both healthcare professionals and organizations reporting PSIs can be confused over the use of the (actual) clinical outcome category. In order to ensure comparability of data, some incidents reported with clinical outcomes of death and significant harm have had to be recoded, as the reporter reported 'potential' rather than 'actual' clinical outcome. There is greater potential for learning in having an option for both types of outcomes. It is recommended that future editions of the NRLS and the ICPS could include categories for both actual and potential harm arising from an incident.

Although the name(s) of medicine involved in the incident are often included as free-form text in the'description of what happened' data field, only $40 \%$ of the medication incidents in 2010 had the name of the medicine in the specified NRLS data field. There are two main reasons for this. Firstly, in some local risk-management reporting systems, both electronic and paper, a separate field to identify the medicine name(s) is not always present. Secondly, the process for local review of medication incidents does not populate the missing data in the medicine name data before submitting the report to the NRLS. Without the name(s) of the medicine recorded in the specified data field, it is difficult to search the incident reports easily and to determine the medicines most frequently associated with incident reports. For the present paper, we have used a manual method to determine the medicines frequently associated with clinical outcomes of death and severe harm.

It is recommended that in future versions of local riskmanagement systems and the NRLS, when reporting a medication incident, the name of the medicine(s) must be included as mandatory data field(s). Medicines names 
should be selected from a national database of medicine products in England. This is currently the Dictionary of Drugs and Devices, published by NHS Connecting for Health [23].

Medication incident reports in the NRLS have variable levels of detail. On average, 89 words are used to describe a severe harm or death incident; however there is a huge range in word count. Significant numbers of incident reports do not include details of 'actions preventing reoccurrence' or 'apparent causes', and this is the case whether or not it relates to severe harm or death. There is limited learning from very short incident reports, with little indication of causes and actions to prevent further incidents. Greater local review and input of additional information from medication incident reports would enable greater learning and system improvement by the local organization and nationally.

It is recommend that sharing anonymized versions of Root Cause Analysis Reports from all NHS organizations and Significant Event Analysis reports from General Practice with the NRLS for all medication incidents reporting death and severe harm would further assist national learning and the spread of safer practice.

\section{The benefits of a national reporting system}

If only small percentages of medication incidents are reported to the NRLS and improvements are required to the data quality of some of the incidents, the question could be asked, 'What are the benefits to patient safety of continuing with this system?' It could be suggested that other systems, such as the Medicines and Health Care Products Regulatory Agency yellow card reporting system for adverse drug reactions, are used instead.

The authors would argue that the NRLS can provide valuable and unique information for learning and system improvement that can be used at both local and national level. When assessing a risk, it is helpful to have data from incidents that have harmed patients and near misses (no harm). It is also essential to have a system that includes an understanding of human factors and patient safety science. Currently, the pharmacovigilence classification systems do not collect near miss data or incident data where a medicine was required but not used (omitted and delayed medicine incidents).

Comparison of the ICPS with pharmacovigilance classification systems such as World Health Authority Adverse Reaction Terminology [24] and Medical Dictionary For Regulatory Activities [25] reveals few human factors and patient-safety science categories in the two pharmacovigilence classifications. Without this type of data and focus, it is difficult to see how the current yellow card reporting system for adverse drug reactions could take on the role for patient safety currently performed by the NRLS.

Furthermore, the NPSA has used a range of approaches to create change and promote safer medication practices. Bottom-up methods include sharing first-hand experience of staff managing medication risks through online webinars and discussion forums. Top-down approaches include introducing purchasing for safety initiatives, where new products/labelling and packaging are requested from healthcare industries, new e-learning modules are made available to practitioners, national designs for patient-held medicines information are developed or new template prescribing, storage, preparation, administration and checking policies are shared.

The NRLS is a unique resource. It has been the information source behind a number of key pieces of advice and guidance for the NHS. Based on the medication incident data reported to the NRLS, together with other data collected from the NHS Litigation Authority, Medical and Healthcare professions protection organizations and published incidents, between 2002 and 2011, the NPSA has published 14 Patient Safety Alerts and Notices, 18 Rapid Response Reports, as well as seven Design for Patient Safety booklets and two themed reviews intended to reduce preventable risks from medicines. Details of all the NPSA medication safety guidance are currently available on the NPSA website [26].

A multimethod independent research study, comprising focus groups and interviews with NHS Chief Pharmacists and an electronic survey of medical, nursing and clinical governance directors, has assessed the quality and impact of the NPSA medication safety outputs [27]. The research concluded that, 'within the NHS, there was a high degree of satisfaction with the medication safety topics addressed which were, with few exceptions, perceived to pose a high risk to patients.'

The medication alerts had stimulated significant work to improve medication safety and was believed by the research participants to have had an important impact on patient safety. Pharmacists welcomed national support for medication safety improvement, despite the resulting workload. Medical Directors were much less likely to be aware of alerts and Rapid Response Reports (RRRs) than their nursing and clinical governance colleagues. One key finding was the inability of around half of NHS trusts to communicate effectively and reliably with their junior doctors.

\section{The future}

Our review has shown the extent of the resource that is the NRLS and specifically, at a granular level, medication PSIs. It has identified those medicines and therapeutic groups most frequently reported in PSIs with clinical outcomes of death and severe harm. The NPSA has issued guidance to help minimize PSIs with many of these medicines. It issued target dates for the NHS to implement its guidance. Yet the NPSA continues to receive serious incident reports involving these medicines, which could have been prevented if the NPSA guidance had been better implemented. 
It is recommended that healthcare organizations in all sectors establish an effective infrastructure to oversee and promote safe medication practice. This should include a multidisciplinary medication safety group. The group should meet monthly to review medication incident report data, improve data quality, and agree and monitor actions intended to minimize risk. This should include tracking progress with the implementation of nationally co-ordinated medication safety guidance. There should be greater transparency on how medication safety is being managed in healthcare organizations. This can be achieved by local organizations publishing an annual medication safety report to interested parties, including the organizations' clinical governance committees, Trust Boards and commissioners.

A number of NHS hospital organizations have appointed medication safety officers (usually pharmacists and sometimes nurses) to provide a focus for medication safety activities in the organization. The benefits of such a post have been described by an academic medical centre in the USA [28], where there is also a Society of Medication Safety Officers [29].

In the future, preventable harms from medication incidents can be further minimized by the continued use of the NRLS to identify and prioritize important actions to improve medication safety, a central organization continuing to issue medication safety guidance to the service and better methods to ensure that the NHS has implemented this guidance. Better implementation could be ensured if healthcare commissioners, the Care Quality Commission, The NHS Litigation Authority and The Welsh Risk Pool required healthcare organizations to provide more detailed evidence that national medication safety guidance has been implemented and demonstrate that there is effective local infrastructure to oversee reporting and learning and to promote safe medication practice.

\section{Competing Interests}

There are no competing interests to declare.

\section{REFERENCES}

1 Department of Health. An organisation with a memory. 2000. Available at http://www.dh.gov.uk/en/Publicationsand statistics/Publications/PublicationsPolicyAndGuidance/ DH_4065083 (last accessed 20 October 2011).

2 Department of Health. Building a safer NHS for patients. Implementing an organisation with a memory. 2001 Available at http://www.dh.gov.uk/en/Publicationsand statistics/Publications/PublicationsPolicyAndGuidance/ DH_4006525 (last accessed 25 September 2011).

3 Department of Health. Liberating the NHS: report of the arms-length bodies review. 2010. Available at
http://www.dh.gov.uk/en/Publicationsandstatistics/ Publications/PublicationsPolicyAndGuidance/DH_117691 (last accessed 25 September 2011).

4 National Patient Safety Agency. Safety in doses. The fourth report from the Patient Safety Observatory. Learning from National Reporting 2005-2006. Available at http://www.nrls. npsa.nhs.uk/resources/patient-safety-topics/medicationsafety/?entryid45=61625 (last accessed 25 September 2011).

5 National Patient Safety Agency. Safety in doses. Improving the safe use of medicines in the NHS. Learning from National Reporting 2007. Available at http://www.nrls. npsa.nhs.uk/resources/patient-safety-topics/medicationsafety/?entryid45=61625 (last accessed 25 September 2011).

6 The NHS Information Centre. Hospital Prescribing England. 2009. Available at http://www.ic.nhs.uk/statistics-and-datacollections/primary-care/prescriptions/hospitalprescribing-england-2009 (last accessed 25 September 2011).

7 The NHS Information Centre. Prescriptions dispensed in the community. England Statistics for 1999 to 2009. Available at http://www.ic.nhs.uk/webfiles/publications/ prescriptionsdispensed/Prescriptions_Dispensed_1999_ 2009\%20.pdf (last accessed 25 September 2011).

8 Dornan T, Ashcroft D, Lewis P, Miles J, Taylor D, Tully MV. An in depth investigation into causes of prescribing errors by foundation trainees in relation to their medical education.EQUIP study. Report to the General Medical Council 2009. Available at http://www.gmc-uk.org/about/ research/research_commissioned_4.asp (last accessed 25 September 2011).

9 Franklin BD, Vincent C, Schachter M, Barber N. The incidence of prescribing errors in hospital inpatients: an overview of the research methods. Drug Saf 2005; 28: 891-900.

10 Stubbs J, Haw C, Taylor D. Prescription errors in psychiatry a multi-centre study. J Psychopharmacol 2006; 4: 553-61.

11 Ghaleb MA, Barber N, Franklin BD, Wong ICK. The incidence and nature of prescribing and medication administration errors in paediatric inpatients. Arch Dis Child 2010; 95: 113-8.

12 James KL, Barlow D, McArtney R, Hiom S, Roberts D, Whittlesea $C$. Incidence, type and causes of dispensing errors: a review of the literature. Int J Pharm Pract 2009; 17: 9-30. Available at http://interruptions.net/literature/ James-IJPP09.pdf (last accessed 25 September 2011).

13 James KL, Barlow D, Burfield R, Hiom S, Roberts D, Whittlesea C. Unprevented or prevented dispensing incidents: which outcome to use in dispensing error research? Int J Pharm Pract 2011; 19: 36-50.

14 James KL, Barlow D, Burfield R, Hiom S, Roberts D, Whittlesea C. Analysis of unprevented dispensing incidents in Welsh NHS hospitals 2003-2004. Int J Pharm Pract 2008; 16: $175-88$.

15 Franklin BD, Jacklin A, Barber N. The impact of an electronic prescribing and administration system on the safety and quality of medication administration. Int J Pharm Pract 2008; 16: 375-9. 
16 Kelly J, Wright D. Medicine administration errors and their severity in secondary care older persons' ward: a multi-centre observational study. J Clin Nurs 2011. doi: 10.1111/j.1365-2702.2011.03760.x. [Epub ahead of print).

17 Ho C, Dean B, Barber N. When do medication administration errors happen to hospital inpatients? Int J Pharm Pract 1997; 5: 91-6.

18 Cavell G, Hughes D. Does computerised prescribing improve the accuracy of drug administration? Pharm J 1997; 259: 782-4.

19 Ridge K, Jenkins D, Noyce P, Barber N. Medication errors during hospital drug rounds. Qual Health Care 1995; 4: 240-3.

20 Barber N, Dean Franklin B, Cornford T, Klecun E, Savage I. Piloting technology evaluations to reduce medication errors. Report to the Patient Safety Research Programme (England). 2005. Available at http://www.haps.bham.ac.uk/ publichealth/psrp/documents/PS019_Final_Report_ Barber.pdf. (last accessed 25 September 2011).

21 Taxis K, Barber N. Ethnographic study of incidence and severity of intravenous drug errors. Br Med J 2003; 326: 684-7.

22 The World Alliance For Patient Safety Drafting Group, Sherman H, Castro G, Fletcher M on behalf of The World Alliance for Patient Safety, Towards an International Classification for Patient Safety: the conceptual framework. Int J Qual Health Care 2009; 21: 2-8. Available at http://intqhc.oxfordjournals.org/content/21/1/

2.full.pdf+html. (last accessed 25 September 2011).

23 NHS Connecting For Health. Dictionary of drugs and devices. Available at http://www.dmd.nhs.uk/about/ index.html. (last accessed 25 September 2011).

24 Uppsala Monitoring Centre. World Health Organisation. Adverse reaction terminology (WHO-ART). Available at http://www.umc-products.com/DynPage.aspx? id $=73589 \& \mathrm{mn} 1=1107 \& \mathrm{mn} 2=1664$. (last accessed 25 September 2011).

25 Medical Dictionary for Regulatory Activities. Maintenance and support services organisation (MedRA MSSO). Available at https://meddramsso.com. (last accessed 25 September 2011).

26 National Patient Safety Agency. Medication Safety Guidance. Patient Safety Resources. Available at http://www.nrls.npsa. nhs.uk/resources/patient-safety-topics/medication-safety (last accessed 25 September 2011).

27 Lankshear A, Lowson K, Weingart SN. An assessment of the quality and impact of NPSA medication safety outputs issued to the NHS in England and Wales. Qual Saf 2011;20: 360-5.

28 Kowiatek R, Weber RJ, Skyedar SJ, Sirio CA. Medication safety manager in an academic medical center. Am J Health-Syst Pharm 2004; 61: 58-64.

29 American Society of Medication Safety Officers. American Society of Medication Safety Officers. Available at http://asmso.org. (last accessed 25 September 2011). 